\title{
A NOVEL SEGMENTATION AND CLASSIFICATION TECHNIQUES FOR MRI BRAIN TUMOR IMAGES
}

\author{
M.P. Abinaya and S.I. Padma \\ Department of Electronics and Communication Engineering, PET Engineering College, India
}

\begin{abstract}
Medical image process is that the most difficult and rising field these days. To solve various problems in medical imaging such as medical image segmentation, object extraction and image classification etc. This work presents a performance of the rough set based approaches. The detection and identification of brain tumour from MRI is crucial to decrease the speed of casualties. Brain tumor is tough to cure, as a result of the brain feature terribly complicated structure and also the tissues are interconnected with one another during a sophisticated manner. The proposed method uses a novel discriminative framework for multilabel automated brain tumor segmentation. The method selects the most relevant features and segments edema and tumor using a classification algorithm based on Multiple Kernel Learning (MKL). Feature selection and dictionary learning in image segmentation are usually combined with RUSBOOST classifier for identifying the tumor. The RF classifier has increased the classification accuracy as evident by quantitative results of our proposed method which are comparable or higher than the state of the art.
\end{abstract}

\section{Keywords:}

Brain Tumor, Segmentation, SVM, MKL, RUSBOOST Algorithm

\section{INTRODUCTION}

Brain tumor is a type of cancers and its death rate is very high. Computer aided images is very good in highlighting and better experiences. Brain tumor problem can be analyzed by separating the tumor tissues such as edema and active tumor from the good tissues. Magnetic Resonance Imaging (MRI) is the technique for brain tumor analysis [1] - [3]. MRI differs from other due to its contrast. MRI protocols is for representing the same object. Segmentation by this method will be quick and exact [15]. Forms, looks and positions of brain tumors are highly dissimilar and the segmentation of brain tumor is a challenging task. In the literature, more number of MRI image has been suggested. Machine learning methods are usually for multi-sequence images [4] [1]. Unsupervised methods [5], doesn't need human action: previously there is no information available on training labels. The example for these methods are the Expectation-Maximization algorithm, the Markov Random Field method and the Gaussian Mixture Model [6] - [9]. In supervised methods [5], the radiologist is set to design a labeled learning set. The specified information by the radiologist is to study a classifier, to labeled person will be classified and segment the total image. Support Vector Machines (SVM) [10], random forests [11] [13] and Bayesian classifier [14] are learning methods.

In the literature, many features can be separated from the brain tumor texture in MR images. These features can be separated into various stages. We distinguish for example statistic measures, intensity based features, texture based features, grey levels, probability based features, wavelets, etc. With this diversity of features, a feature selection step implemented separately is often necessary to eliminate the redundancy and to select the most useful features using a discrimination power criterion [16] or a class separability criteria [18].

In Multiple Kernel Learning (MKL) [17], the classification and the feature selection are done in the same optimization problem. The idea of the MKL is to select one or more kernel functions to each feature [19]. For each of these functions, a positive weight can be associated. This weight reflects the importance of the corresponding kernel (feature) within the classification [22]-[26]. The kernel weight increases if the corresponding kernel (feature) is judged informative and decreases if it is judged less informative. A thin constraint is applied on the kernel weights to force a number of them to be up to zero. The corresponding features are considered as noninformative features and are not computed during the test step [27]-[29].

Brain tumor segmentation is based on MRI multi-sequence. The proposed method is based on classification using MKL algorithm to exploit the diversity and the complementarity of the data supplied by the different images [20]. From every kind of pictures, we tend to reckon an outsized set of options from a little variety of voxels hand-picked by associate the degree of knowledgeable to make a coaching feature base [21]. In the learning step, the most informative options from the feature base are hand-picked.

\section{METHOD}

This method is classified into three: a learning step, a classification step and a post-processing step. In this paper, we present a brain tumor segmentation method from MRI multisequence. This method uses a MKL algorithm for different types of images. From this method, predict the texture and tumor present in the image. In the learning step, the specific content is chosen from the given image. During testing, chosen images are taken to find the tumor and edema. A post-processing is used to improve the output. This method focusses mainly on segmentation and classification. The architecture of which is given in Fig.1.

\subsection{TRAINING AND TESTING DATASET}

The training and testing images are taken from BRATS 2013 Dataset. This dataset includes High-Grade Glioma patients (HG: advanced stages) and Low-Grade Glioma patients (LG: first stages). The segmentation problem is a multiclass problem: from the patient, we have to segment edema tissues, tumor tissues and healthy tissues. All MRI images are inserted to $1 \mathrm{~mm}$ isotropic resolution.

The MR images are provided with the corresponding reference and segmentations. The ground truths were built by merging the 
annotations of the different experts for edema and tumor. As features are extracted from multiple images and multiple MRI sequences, we need to use multiple kernel function, instead of a unique kernel, to fully exploit the diversity and the known complementarity of MRI sequences.

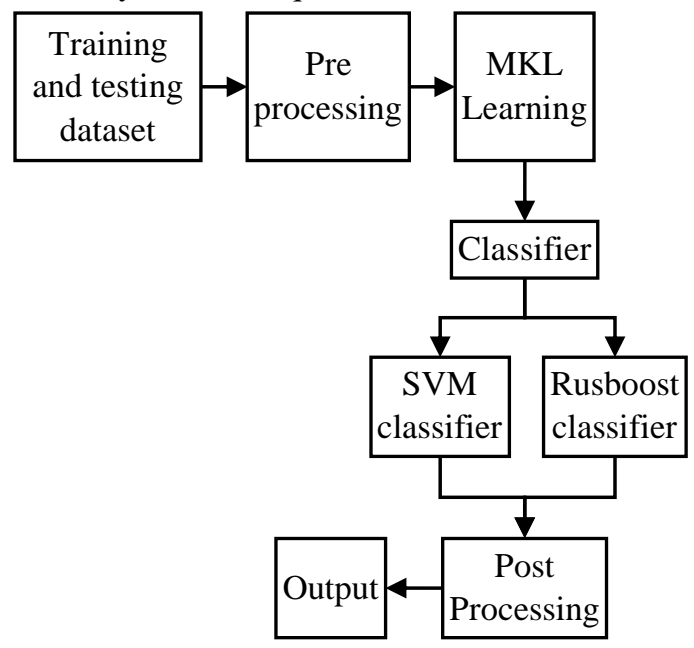

Fig.1. Block Diagram

\subsection{PRE-PROCESSING}

The main importance of this method is to remove the noise present in the image. Guided filter is used in this method for edge preserving, smoothing and structure transferring filtering.

If pre-processing is to correct some downgrading in the image, the nature of special information is: $v$ is the downgrading function; only few properties of downgrading will be assumed. Knowledge on $v$ about this image acquisition was obtained.

\subsection{KERNEL DICTIONARY LEARNING}

The purpose of the learning step is to validate the different parameters of the method. The learning stage is divided into three steps: the labeling step, the feature extraction step and the MKLSVM learning step. The labeling step is computed as follow: For each patient, the expert is asked to choose one slice from a single sequence and to select points in the border of edema, in the border of tumor and in healthy tissues. From these points, we can draw the boundaries of the different tissues and generate later different training sets.

For each training set, different features can be extracted such as intensity based features, texture based features and wavelets. The associated feature matrix is computed: for each voxel, different features are concatenated in a feature vector. All vectors provide the learning feature matrix. The purpose of the MKLSVM learning is to solve the MKL-SVM problem (SVM parameters and weights $d_{m}$ ) and to validate its regularization parameter $C$ based on the training labeled voxels. SVM parameters and kernel weights are adjusted automatically by the algorithm. The regularisation parameter $\mathrm{C}$ must be fixed by us. For that reason, we adopted a simple and fully automatic procedure to validate $C$ : we initialize $C$ with a very small value and increment regularly. Increasing $C$ results increasing the tumor region and edema region. From a certain value of $C$, these two regions become stable. The corresponding kernel weights become also stable. The value of $C$ is chosen when the variation of kernel weights becomes lower than a given threshold.

The multiple kernel learning method has been developed to determine the positive weight $d_{m}$ associated to each kernel $k_{m}$. The associated decision function has the following form, as in Eq.(1)

$$
f(x)=\sum_{m=1}^{M} f_{m}(x)=\sum_{i=1}^{n} \alpha_{i} y_{i} \sum_{m=1}^{M} d_{m} k_{m}\left(x, x_{i}\right)
$$

\subsection{SVM CLASSIFICATION}

The classification process is done over the learned features. SVM classifier is applied, features and the classification is done. Once the value of $\mathrm{C}$ is chosen and the MKL model is learned, the chosen features are computed for the whole test voxels, the corresponding kernels are associated and the decision function is applied to classify the different voxels. We can associate one or more kernel functions. Each kernel is associated with a positive coefficient reflecting the importance of the corresponding kernel (feature) in the classification. The initial classification result includes always errors.

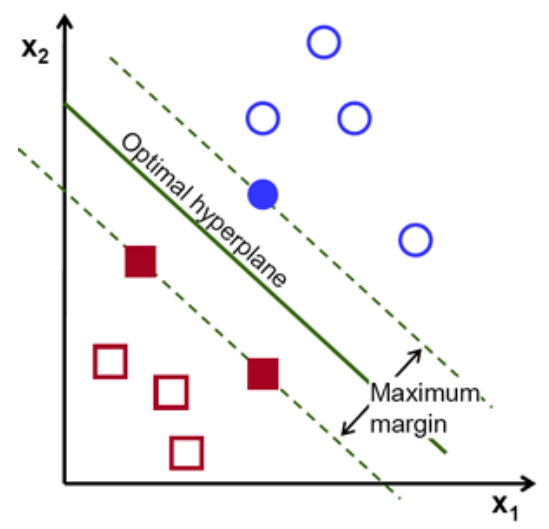

Fig.2. SVM Working Model

Step 1: The idea of support vector machine is to create a hyper plane in between data sets to indicate which class it belongs to.

Step 2: The feature vector is given as input to the classifier.

Step 3: The feature vectors of the database images are divided into training and testing vectors.

Step 4: The classifier trains on the training set and applies it to classify the testing set.

Step 5: The performance of the classifier is measured by comparing the predicted labels and actual values.

\subsection{RUSBOOST CLASSIFIER}

The RUSBOOST algorithm is used for improving the performance of the trained data set acquired from the skewed data set. A hybrid sampling/boosting algorithm called RUSBOOST algorithm. It is simple and fast technique to combine boosting and sampling data. RUS is a technique that eliminate the data distribution imbalances between the classes and improve the classification performance of the weak classifiers. Boosting can be performed by both re-weighting and resampling. In this method, re-sampling is performed according to the weights given to samples included in the training data set. 
The RUSBOOST algorithm is used for improving the performance of the trained data set acquired from the skewed data set. A hybrid sampling or boosting algorithm called RUSBOOST algorithm. It is simple and fast technique to combine boosting and sampling data. RUS is a technique that eliminate the data distribution imbalances between the classes and improve the classification performance of the weak classifiers. Boosting can be performed by both re-weighting and resampling. In this method, re-sampling is performed according to the weights given to samples included in the training data set.

\subsection{POST PROCESSING}

The purpose of the post-processing step is to improve the classification result. Very small regions are eliminated using morphological operations and a re-application of our MKL-SVM algorithm on a reduced area around the first segmentation result is done. New segmentations for the tumor and the edema are then obtained. A combination of both classification results was done to obtain final segmentation as done. Median filter is used for post processing. The median filter is an efficient methodology which will, to some extent, distinguish out-of-range isolated noise from legitimate image features such as edges and lines.

The median filter can be expressed as,

$$
y[m, n]=\operatorname{median}\{x[i, j], \text { where }(i, j) \in w\}
$$

Specifically, the median filter replaces an element by the median, rather than the typical, of all pixels during a neighborhood.

\section{RESULT AND DISCUSSION}

\subsection{BRAIN TUMOR DETECTION USING SVM ALGORITHM}

The training and testing images are taken from BRATS 2013 Dataset. In second step preprocessing is used. The objective of the preprocessing phase is to apply possible image enhancement techniques to obtain the required visual quality of the images. In the proposed method guided filter is used for pre-processing. Next step, edge detection is used. Edge detection can be defined as the discovery of lines that marks the limit and divides of image appearance from other places or things in a digital image. Image segmentation is the process of partitioning a digital image into multiple segments. Super-pixel segmentation is dividing an image into hundreds of non-overlapping super-pixels.

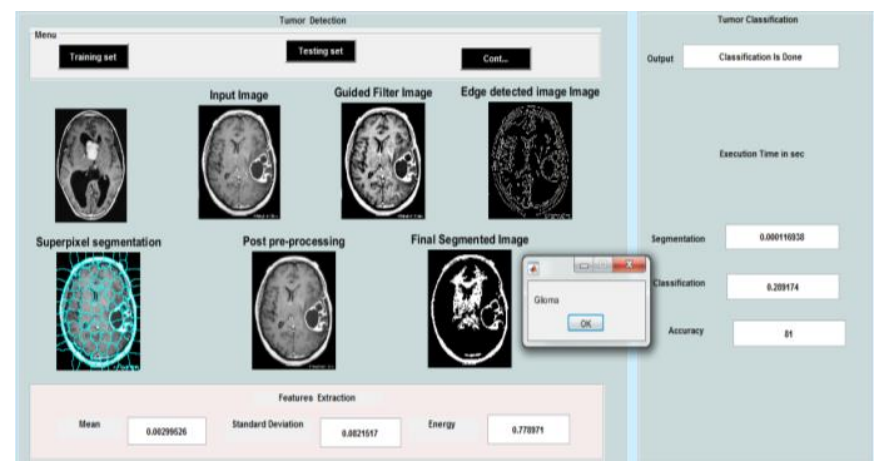

Fig.3. Tumor Image using SVM classifier
In final step post preprocessing is used. The purpose of the post-processing step is to improve the classification result. Median filter is used for post processing. In final stage, the SVM classifier is used for classification. The final tumor detected segmentation image is shown in Fig.3. The output will be displayed on the message box.

The stages of Feature selection and classification using Multiple Kernel learning for brain tumor segmentation using SVM classifier will be explained below.

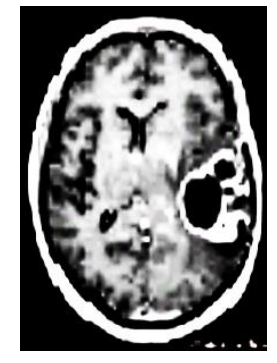

Fig.4. Input image

The training and testing images are taken from BRATS 2013 Dataset. Training and testing images consists of high grade (advance stage) and low grade (initial stage) of glioma taken from the patients. Training images are in build and the testing images are used for the following stages.

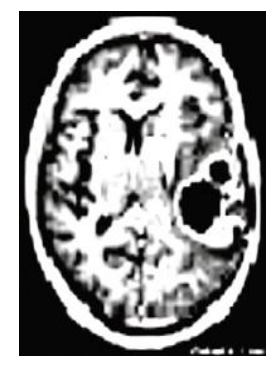

Fig.5. Guided filter image

In the proposed method guided filter is used for preprocessing. The aim of pre-processing is an improvement of the image data that suppresses unwanted distortions or enhances some image features important for further processing.

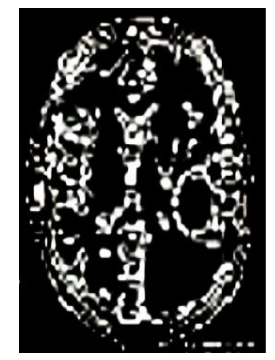

Fig.6. Edge detected image

Edge detection can be defined as the discovery of lines that marks the limit and divides of image appearance from other places or things in a digital image. Canny edge detection algorithm is used in edge detection. The Canny edge detector is an edge detection operator that uses a multi-stage algorithm to detect a wide range of edges in images. 


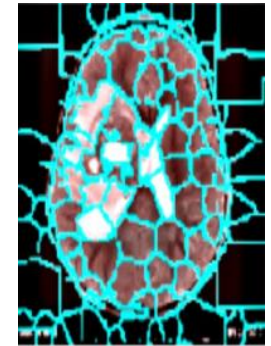

Fig.7. Super-pixel segmentation

Image segmentation is the process of partitioning a digital image into multiple segments. Super-pixel segmentation is dividing an image into hundreds of non-overlapping super-pixels. Super pixel segmentation algorithm is used in image segmentation. Image segmentation is typically used to locate objects and boundaries (lines, curve etc.) in images.

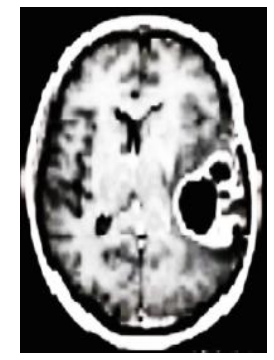

Fig.8. Post-processing

The purpose of the post-processing step is to improve the classification result. Median filter is used for post processing. Median filter is used to remove noise from an image. The aim of pre-processing is an improvement of the image data that suppresses unwanted distortions or enhances some image features important for further processing.

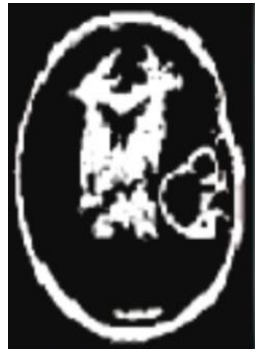

Fig.9. Final Output

The output will be displayed on the message box. The types of diseases and the accuracy will be displayed on the message box.

\subsection{BRAIN TUMOR DETECTION USING RUSBOOST ALGORITHM}

The training and testing images are taken from BRATS 2013 Dataset. In second step preprocessing is used. The objective of the preprocessing phase is to apply possible image enhancement techniques to obtain the required visual quality of the images. In the proposed method guided filter is used for pre-processing. Next step, edge detection is used.
Edge detection can be defined as the discovery of lines that marks the limit and divides of image appearance from other places or things in a digital image. Image segmentation is the process of partitioning a digital image into multiple segments. Super-pixel segmentation is dividing an image into hundreds of nonoverlapping super-pixels.

In final step post preprocessing is used. The purpose of the post-processing step is to improve the classification result. Median filter is used for post processing. In final stage, the RUSBOOST classifier is used for classification. The output will be displayed on the message box. Below figure shows the type of tumor and its accuracy.

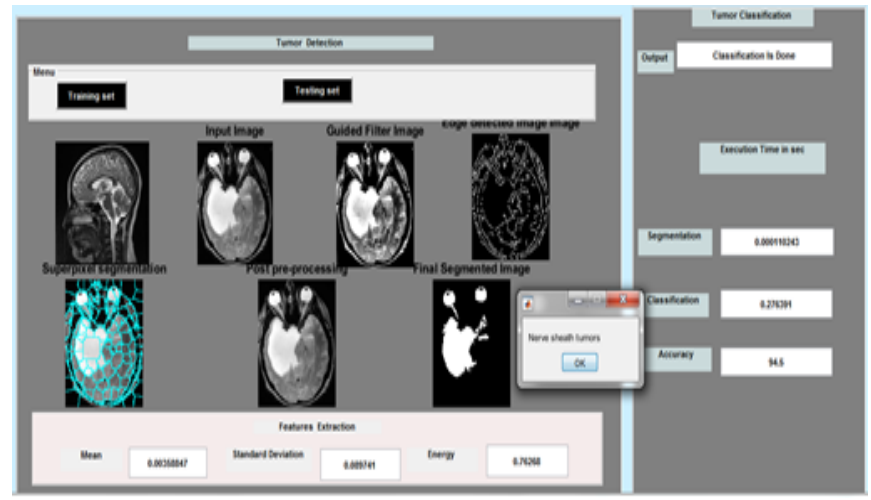

Fig.10. Tumor Image using RUSBOOST classifier

BRATS 2013 dataset is used for both training and testing data. Training and testing images consists of high grade (Advance stage) and low grade (initial stage) of Glioma taken from the patients.

The stages of Feature selection and classification using Multiple Kernel learning for brain tumor segmentation using RUSBOOST classifier will be explained below.

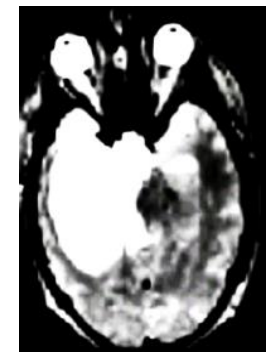

Fig.11. Input Image

In the pre-processing stage guided filter is used. The guided filter is used to remove noise. The pre-processing is used to suppresses unwanted distortions and improve the visual quality of image.

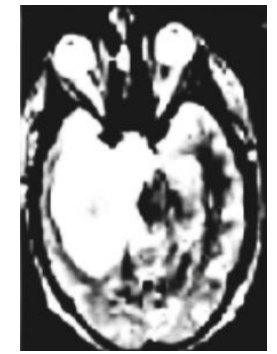

Fig.12. Guided Filter 
The sudden changes of discontinuities in an image are called as edges. Edge detection can be defined as the discovery of lines that marks the limit and divides of image appearance from other places or things in a digital image. Canny edge detection algorithm is used in edge detection.

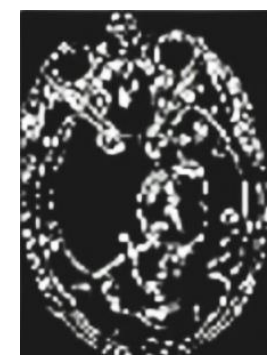

Fig.13. Edge Detection

Super - pixel segmentation is dividing an image into hundreds of non-overlapping super-pixels. It is used to locate objects and boundaries in images.

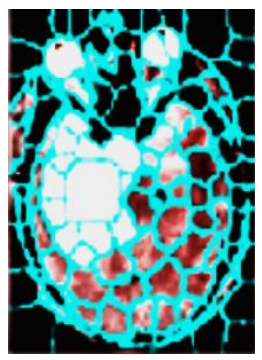

Fig.14. Super-pixel Segmentation

The post-processing step is to improve the classification result. Median filter is used for post processing. Median filter is used to remove noise from an image.

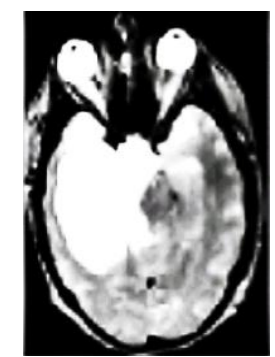

Fig.15. Post-processing

The Final Output consists of types of diseases, accuracy, Mean and Standard deviation will be displayed on the message box.

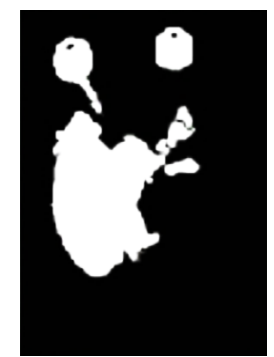

Fig.16. Final Output
The Final Output consists of types of diseases, accuracy, Mean and Standard deviation will be displayed on the message box.

\subsection{PERFORMANCE ANALYSIS OF SVM AND RUSBOOST CLASSIFIER}

Brain tumor detection using SVM and RUSBOOST algorithm are compared in terms of accuracy are shown below. The accuracy value of RUSBOOST will be improved compared with the SVM Classifier. The classifier in which the RUSBOOST classifier shows the good result.

Table.1. Performance analysis of SVM and RUSBOOST Algorithm

\begin{tabular}{|l|c|c|}
\hline \multicolumn{1}{|c|}{ Parameters } & $\begin{array}{c}\text { SVM } \\
\text { Classifier }\end{array}$ & $\begin{array}{c}\text { RUSBOOST } \\
\text { Classifier }\end{array}$ \\
\hline Accuracy & 81 & 94.5 \\
\hline Mean & 0.0029 & 0.0035 \\
\hline Standard deviation & 0.0821 & 0.0897 \\
\hline Energy & 0.7 & 0.7 \\
\hline
\end{tabular}

\section{CONCLUSIONS}

The proposed system has been developed to diagnosis of brain tumor from Magnetic Resonance Imaging (MRI) of the brain. There are many phases to detect the tumor part from the brain image and type of tumor. Segmentation extracts the tumor portion. Median filter is used for noise removal. The method takes place in three stages; a learning step, a classification step and a post processing step. The method uses a multiple kernel learning algorithm allowing to do the feature selection and the classification in a unique optimization problem. It consists of selecting the features that optimize the classification. In the proposed system SVM and RUSBOOST classifiers are used as classification algorithm. In Existing method, the SVM algorithm is used. The classification accuracy of RUSBOOST classifier is increased in proposed system. RUSBOOST presents a simpler, faster, and less complex alternative to other methods. In future work, we tend to enrich our feature base by adding alternative classic and new options.

\section{REFERENCES}

[1] S. Bauer, R. Wiest, L.P. Nolte and M. Reyes, "A Survey of MRI-based Medical Image Analysis for Brain Tumor Studies", Physics in Medicine and Biology, Vol. 58, No. 13, pp. 97-104, 2013.

[2] N. Gordillo, E. Montseny and P. Sobrevilla, "State of the Art Survey on MRI Brain Tumor Segmentation", Magnetic Resonance Imaging, Vol. 31, No. 8, pp. 1426-1438, 2013.

[3] E.S.A. El-Dahshan, H.M. Mohsen, K. Revett and A.B.M. Salem, "Computer-Aided Diagnosis of human Brain Tumor through MRI: A Survey and A New Algorithm", Expert systems with Applications, Vol. 41, No. 11, pp. 5526-5545, 2014.

[4] L.R. Schad, S. Bluml and I. Zuna, "Ix. MR Tissue Characterization of Intracranial Tumors by means of 
Texture Analysis", Magnetic Resonance Imaging, Vol. 11, No. 6, pp. 889-896, 1993.

[5] T. Hastie, R. Tibshirani and J. Friedman, "The Elements of Statistical Learning", Springer, 2001.

[6] M. Farzinfar, E.K. Teoh and Z. Xue, "A Joint Shape Evolution Approach to Medical Image Segmentation using Expectation-Maximization Algorithm", Magnetic Resonance Imaging, Vol. 29, No. 9, pp. 1255-1266, 2011.

[7] T. Zhang, Y. Xia and D.D. Feng, "Hidden Markov Random Field Model based Brain MR Image Segmentation using Clonal Selection Algorithm and Markov Chain Monte Carlo Method", Biomedical Signal Processing and Control, Vol. 12, No. 1, pp. 10-18, 2014.

[8] Z. Ji, Y. Xia, Q. Sun, Q. Chen and D. Feng, “Adaptive Scale Fuzzy Local Gaussian Mixture Model for Brain MR Image Segmentation", Neuro Computing, Vol. 134, pp. 60-69, 2014.

[9] F. Dong and J. Peng, "Brain MR Image Segmentation based on Local Gaussian Mixture Model and Nonlocal Spatial Regularization", Journal of Visual Communication and Image Representation, Vol. 25, No. 5, pp. 827-839, 2014.

[10] S. Bauer, L.P. Nolte and M. Reyes, "Fully Automatic Segmentation of Brain Tumor Images using Support Vector Machine Classification in Combination with Hierarchical Conditional Random Field Regularization", Proceedings of International Conference on Medical Image Computing and Computer Assisted Intervention, pp. 354-361, 2011.

[11] D. Zikic, B. Glocker, E. Konukoglu, A. Criminisi, C. Demiralp, J. Shotton, O. Thomas, T. Das, R. Jena, and S. Price, "Decision Forests for Tissue-Specific Segmentation of High-Grade Gliomas in multi-Channel MR", Proceedings of International Conference on Medical Image Computing and Computer-Assisted Intervention, pp. 369-376, 2012.

[12] E. Geremia, O. Clatz, B.H. Menze, E. Konukoglu, A. Criminisi, and N. Ayache, "Spatial Decision Forests for MS Lesion Segmentation in Multichannel Magnetic Resonance Images", Neuroimage, Vol. 57, No. 2, pp. 378-390, 2011.

[13] J.J. Corso, E. Sharon, S. Dube, S. El-Saden, U. Sinha and A. Yuille, "Efficient Multilevel Brain Tumor Segmentation with Integrated Bayesian Model Classification", IEEE Transactions on Medical Imaging, Vol. 27, No. 5, pp. 629640, 2008

[14] B.H. Menze, A. Jakab, S. Bauer, J. Kalpathy-Cramer, K. Farahani, J. Kirby, Y. Burren, N. Porz, J. Slotboom and R. Wiest, "The Multimodal Brain Tumor Image Segmentation Benchmark", IEEE Transactions on Medical Imaging, Vol. 34, No. 10, pp. 1993-2024, 2015.

[15] A. Ladgham, A. Sakly and A. Mtibaa, "MRI brain tumor recognition using Modified Shuffled Frog Leaping Algorithm", Proceedings of $15^{\text {th }}$ International Conference on Sciences and Techniques of Automatic Control and Computer Engineering, pp. 504-507, 2014.

[16] G.R. Lanckriet, N. Cristianini, P. Bartlett, L.E. Ghaoui and M.I. Jordan, "Learning the Kernel Matrix with Semi Definite Programming", Journal of Machine Learning Research, Vol. 5, No. 2, pp. 27-72, 2004.
[17] He Lin, Qianyi Wang, Xin Lu and Haibo Li, "Hybrid MultiGranulation Rough Sets of Variable Precision Based on Tolerance", Proceedings of $12^{\text {th }}$ International Conference on Fuzzy Systems and Knowledge Discovery, pp. 231-235, 2015.

[18] P. Vivekanandan, "An efficient SVM based tumor classification with symmetry non-negative matrix factorization using gene expression data", Proceedings of International Conference on Information Communication and Embedded Systems, pp. 761-768, 2013.

[19] L. Wang, "Feature Selection with Kernel Class Separability", IEEE Transactions on Pattern Analysis and Machine Intelligence, Vol. 30, No. 9, pp. 1534-1546, 2008.

[20] M. Farzinfar, E.K. Teoh and Z. Xue, "A Joint Shape Evolution Approach to Medical Image Segmentation using Expectation-Maximization Algorithm", Magnetic Resonance Imaging, Vol. 29, No. 9, pp. 1255-1266, 2011.

[21] M. Fernandez Delgado, E. Cernadas, S. Barro and D. Amorim, "Do We Need Hundreds of Classifiers to Solve Real World Classification Problems", Journal of Machine Learning Research, Vol. 15, No. 1, pp. 3133-3181, 2014.

[22] M.K. Abd-Ellah, A.I. Awad, A.A.M. Khalaf and H.F.A. Hamed, "Design and Implementation of a Computer-Aided Diagnosis System for Brain Tumor Classification", Proceedings of $28^{\text {th }}$ International Conference on Microelectronics, pp. 73-76, 2016.

[23] M. Liu, X. Zhang and B. Wang, "Research on Agricultural Decision Support System based on Rough Set Theory", Proceedings of International Conference on Future BioMedical Information Engineering, pp. 102-109, 2009.

[24] N. Gordillo, E. Montseny and P. Sobrevilla, "State of the Art Survey on MRI Brain Tumor Segmentation", Magnetic Resonance Imaging, Vol. 31, No. 8, pp. 1426-1438, 2013.

[25] N. Boughattas, M. Berar, K. Hamrouni and S. Ruan, "A ReLearning based Post-Processing Step for Brain Tumor Segmentation from Multi Sequence Images", International Journal of Image Processing, Vol. 10, No. 2, pp. 50-62, 2016.

[26] Q. Kang, H. He, H. Wang and C. Jiang, "A Novel Discrete Particle Swarm Optimization Algorithm for Job Scheduling in Grids", Proceedings of $4^{\text {th }}$ International Conference on Natural Computation, pp. 401-405, 2008.

[27] R. Diao, S. Jin and Q. Shen, "Antecedent Selection in Fuzzy Rule Interpolation using Feature Selection Techniques", Proceedings of IEEE International Conference on Fuzzy Systems, pp. 2206-2213, 2014.

[28] R. Jensen and Q. Shen, "Semantics-Preserving Dimensionality Reduction: Rough and Fuzzy-Rough-Based Approaches", IEEE Transactions on Knowledge and Data Engineering, Vol. 16, No. 12, pp. 1457-1471, 2004.

[29] R. Li, "Medical Image Segmentation Based on Watershed Transformation and Rough Sets", Proceedings of $4^{\text {th }}$ International Conference on Bioinformatics and Biomedical Engineering, pp. 1-5, 2010. 